\title{
Synthesis and Spectral Studies of Ru(II) complexes with Macrocyclic Ligands
}

\author{
ASHISH RAJAK ${ }^{1 \#, ~ A R P I T ~ S R I V A S T A V A ~}{ }^{1}$, RAMAKANT'1, SUBHASH CHANDRA \\ SHRIVASTAVA ${ }^{1 *}$, RANJEET SINGH CHAUHAN ${ }^{2}$, UDAY SINGH PATEL ${ }^{3}$ and \\ SHEKHAR SRIVASTAVA ${ }^{1}$
}

1Department of Chemistry, University of Allahabad, Prayagraj-211002 UP, India.

'Department of Chemistry, Gochar Mahavidyalaya, Rampur Maniharan, Saharanpur-247451 UP, India.

${ }^{3}$ Department of Chemistry, D A V (P G) College Knapur-208001 UP, India.

*Corresponding author E-mail: subhash.chem.au@gmail.com, kumarashish8574@gmail.com

http://dx.doi.org/10.13005/ojc/370401

(Received: June 26, 2021; Accepted: July 27, 2021)

\begin{abstract}
Fifteen $\mathrm{Ru}(\mathrm{II})$ complexes of the type $\left[\mathrm{RuCl}_{2}(\mathrm{~L})\right]$ (where $\mathrm{L}=\mathrm{N}_{4}$ donor macrocyclic ligands) have been synthesised by reaction of $\left[\left(\mathrm{RuCl}_{2}(\mathrm{DMSO})_{4}\right]\right.$ with fifteen macrocyclic Schiff base ligands containing $\mathrm{N}_{4}$ donors groups. The prepared fifteen $\left[\mathrm{RuCl}_{2}(\mathrm{~L})\right]$ complexes were characterised by elemental analyses, molar conductivity, UV-Visible, IR, X-ray photoelectron spectra (XPS) and magnetic susceptibility measurements and an octahedral geometry was proposed for all these complexes.
\end{abstract}

Keywords: $\mathrm{N}_{4}$ macrocyclic shiff base complexes, $\mathrm{Ru}$ (II) complexes, elemental analysis, UV-Visible, Magnetic susceptibility measurement, X-ray photoelectron spectra (XPS).

\section{INTRODUCTION}

During past few decades the chemistry of macrocyclic of metal have been developed very extensively due to its applications in coordination chemistry $^{1,2}$; bioinorganic chemistry ${ }^{3}$ catalysis ${ }^{4-6}$; organometallic chemistry ${ }^{7}$ and biocoordination chemistry $^{8}$. Many macrocyclic ligands e-g. Robson type tetraiminodiphenol macrocyclic ligands ${ }^{9,10}$; $\mathrm{N}_{4} \mathrm{~S}_{2}$ donor macrocyclic ligands ${ }^{11}$; crown ethers; porphyrins; saturated and unsaturated polyamine 6-12; polyazamacrocycles ${ }^{18}$; have been synthesised.
A number of schiff base macrocyclic ligands have been reported ${ }^{14}$. In which many macrocyclic ligands have been synthesised by the template condensation reaction ${ }^{15}$. Several transition metal ion complexes in living organisms work as enzymes or carriers in macrocyclic ligand environment ${ }^{16}$ and used as modelling on the active sites of metalloenzymes ${ }^{17}$.

Various macrocyclic metal complexes have been used as detecting tumor lesions ${ }^{18}$ as in labeling monoclonal antibodies with radioactive models ${ }^{19}$ as in

This is an Open Access article licensed under a Creative Commons license: Attribution 4.0 International (CC- BY). Published by Oriental Scientific Publishing Company @ 2018 
metal ion separation ${ }^{20}$ as anticancerous ${ }^{21}$ as toxicity against bacterial fungal growth ${ }^{22}$ as photosenstizer ${ }^{23}$; in photosynthesis and dioxygen transport ${ }^{24}$; catalyst ${ }^{25-27}$ as pharmalogical agent; ${ }^{28,29}$ cancer diagonsis $^{30-32}$ as therapeutic radiotherapeutic ${ }^{33}$ as antitumour ${ }^{34}$ as potential medical applications ${ }^{35}$ as environmental importance ${ }^{36}$ as RNA cleavage catalyst $^{37}$ and as NMR shift and relaxation agents ${ }^{38}$.

Few comprehensive reviews on macrocyclic ligands, their metal complexes and applications have been also published in last few years ${ }^{39}$.

Many ruthenium (II) \& (III) Schiff base and macrocyclic Schiff base complexes have been extensively studied in last few years due to their importance as biochemical and analytical reagents ${ }^{40}$. But ruthenium (II) macrocyclic Schiff base complexes are less known e.g. Spectroscopic data studies and structures of trans-Ru(II) and $\mathrm{Ru}$ (III) bis (cyanide) complexes sustained by tetradentate macrocyclic tertiary amine ligand ${ }^{41}$; $\mathrm{N}$-macrocyclic complexes of $\mathrm{Ru}(\mathrm{II})$ \& $\mathrm{Ru}(\mathrm{III})$; tetraaza macrocyclic $R u(I I)$ complexes: synthesis, spectral and catalytic studies ${ }^{42}$; the versatile $\mathrm{Ru} I \mathrm{I} / \mathrm{Ru}$ III teraaza macrocyclic complexes and their nitrosyl derivative ${ }^{43}$; Ru(II) \& (III) complexes with cyclam and related species ${ }^{44} ; R u(I I) /(I I I)$ and oxovanadiun (IV) complexes with macrocyclic schiff base complexes; $\mathrm{Ru}(\mathrm{II})$ complexes of a new tetrapyrazolic macrocyclic; some new polynucleating ligands containing $R u(I I)$ complexes incorporating terpyridyl and macrocyclic aza-crown binding sites. Recently some reviews on macrocyclic ligands have been also published ${ }^{45}$.

Shankar et al., ${ }^{46}$ have been synthesised various ruthenium (II) complexes with macrocyclic Schiff base ligands derived from orthophthaldehyde and different diamines. Characterised and used as catalysts for the reduction of pralidoxime to its amino derivatives.

In this present communication, fifteen macrocyclic schiff base ligands will be synthesie by condensation reaction of 2,5-diformyl furan or furil or phenanthrene 9,10-dione with different aliphatic diamines $\mathrm{NH}_{2}\left(\mathrm{CH}_{2}\right) \mathrm{nNH}_{2}$ i.e. $\mathrm{NH}_{2} \mathrm{CH}_{2} \mathrm{CH}_{2} \mathrm{NH}_{2}$ or $\mathrm{NH}_{2} \mathrm{CH}_{2} \mathrm{CH}_{2} \mathrm{CH}_{2} \mathrm{NH}_{2}$ or $\mathrm{NH}_{2} \mathrm{CH}_{2} \mathrm{CH}_{2} \mathrm{CH}_{2} \mathrm{CH}_{2} \mathrm{NH}_{2}$ or $\mathrm{NH}_{2} \mathrm{CH}_{2} \mathrm{CH}_{2} \mathrm{CH}_{2} \mathrm{CH}_{2} \mathrm{CH}_{2} \mathrm{NH}_{2}$ or $\mathrm{NH}_{2} \mathrm{CH}_{2} \mathrm{CH}_{2} \mathrm{CH}_{2}$ $\mathrm{CH}_{2} \mathrm{CH}_{2} \mathrm{CH}_{2} \mathrm{NH}_{2}$ and their ruthenium(II) metal complex through template synthesis in 2:2:1 molar ratio respectively.

\section{EXPERIMENTAL}

$\mathrm{RuCl}_{3} \cdot 3 \mathrm{H}_{2} \mathrm{O}$ (Johnson Matthey \& Co, Ltd; triphenylphosphine Merck, Mumbai); ethanol (Merck, Mumbai); dichloromethane (Aldrich) and acetone (Aldrich) were AR Grade and used after purification and dried. Solvents were distilled from relevent drying agents immediately in advance of use. $\left[\mathrm{RuCl}_{2}\right.$ $\left.\left(\mathrm{PPh}_{3}\right)_{4}\right]$ was prepared by published method.

Melting points were determined by using in sealed capillary tube on Melting point apparatus. The $\mathrm{C}$ and $\mathrm{H}$ were determined by CDRI, LUCKNOW, INDIA. Nitrogen and chlorine were determined by Kjeldahl's and Volhard's methos respectively. Molar conductivity was measured on Elico-CM 82 conductivity bridge in acetone at room temperature. IR spectra were recorded on Perkin-Elmer 1000 IR spectrometer using KBr/CsI pellets. Electronic spectral measurements were recorded on Elico SL159 spectrophotometer in the range $300-1000 \mathrm{~nm}$. Magnetic measurements were carrid out a Cahn 2000 electo balance by Faraday Method using $\mathrm{Hg}\left[\mathrm{Co}(\mathrm{SCN})_{4}\right]$ as calibrant. Pascal constants were used for diamagnetic corrections. Kratos analytical Axis Supra ESCA i.e. X-ray photoelectron spectroscopy i.e. XPS instrument equipped with monochromatised Alka $(1486.6 \mathrm{eV})$ source is used. All the peaks were rectified for charging with reference to $\mathrm{C} 1 \mathrm{~S}$ peak $284.8 \mathrm{eV}$ and counterd with Shirley background and a union of Gavssian and Lorentzian lineshapes, using ESCApe software.

\section{Prepration of $\left[\mathrm{RuCl}_{2} \cdot \mathrm{L}^{1-15}\right]$ complexes}

2,5- diformylfuran or furil or phenanthrene 9,10-dione was mixed in dry ethanolic solution of $\mathrm{NH}_{2}\left(\mathrm{CH}_{2}\right)_{\mathrm{n}} \mathrm{NH}_{2}(2 \mathrm{mmol})$ (where $(2 \mathrm{mmol}) \mathrm{n}=2$ or 3 or 4 or 5 or 6 ) and refluxed for $2 \mathrm{~h}$ for preparation of ligands $L_{1}$ to $L_{15}$ ). In this resulting each solution poured $\left[\mathrm{RuCl}_{2}\left(\mathrm{PPh}_{3}\right)_{4}(1 \mathrm{mmol})\right.$ i.e. 1:1 molar ratio and again refluxed for about 2 hours. The resulting precipitate was filtered and recrystallised by benzene: pet-ether (9:1) ratio (Figures 1-3). 


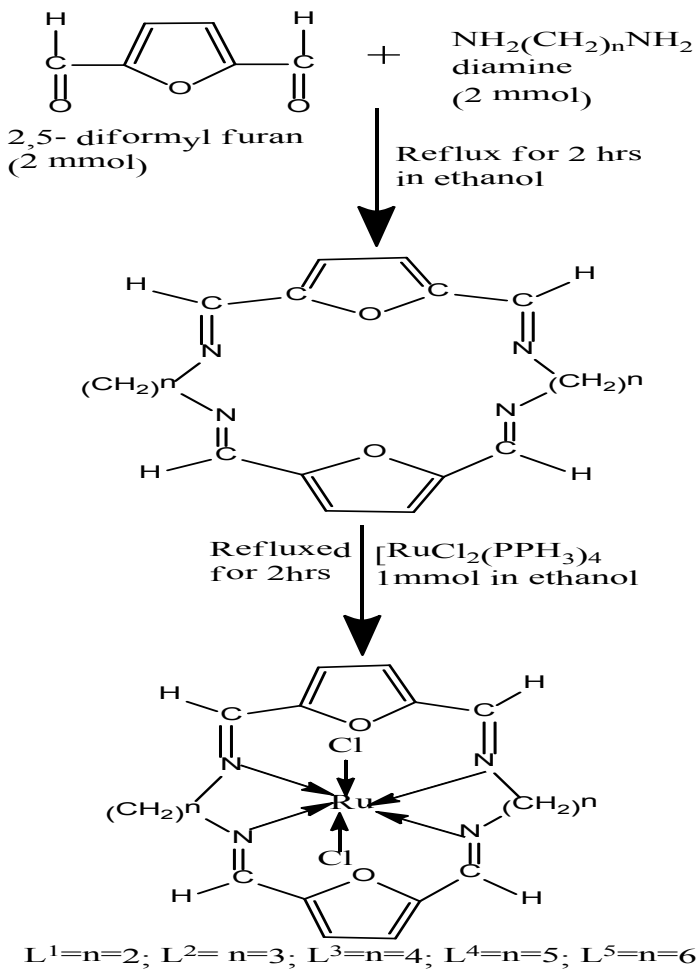

Fig. 1. Preparation of $\left[\mathrm{RuCl}_{2} \mathrm{~L}^{1-5}\right]$ complexes

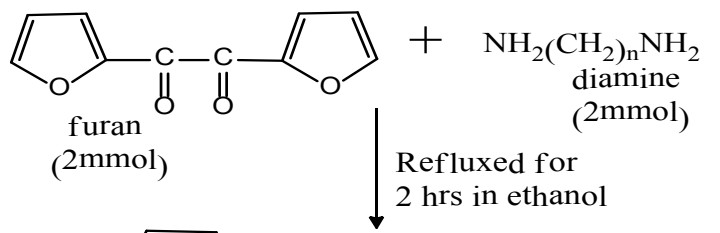<smiles>CN=C(c1nc(-c2ccco2)n[15c](-c2ccco2)n1)c1ccco1</smiles>

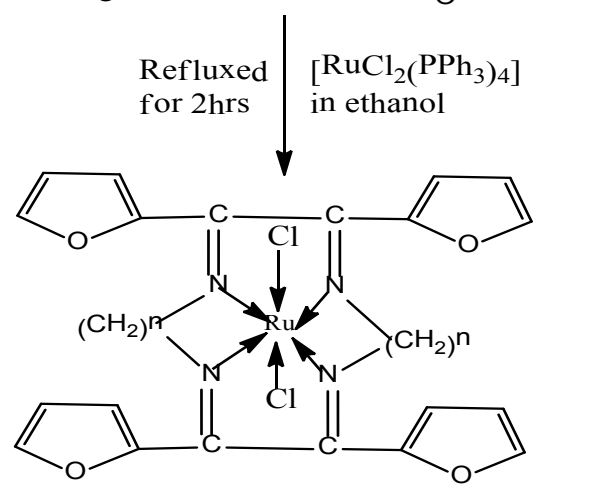

$\mathrm{L}^{6}=\mathrm{n}=2 ; \mathrm{L}^{7}=\mathrm{n}=3 ; \mathrm{L}^{8}=\mathrm{n}=4 ; \mathrm{L}^{9}=\mathrm{n}=5 ; \mathrm{L}^{10}=\mathrm{n}=6$

Fig. 2. preparation of $\left[\mathrm{RuCl}_{2}\left(\mathrm{~L}^{6-10}\right)\right]$ complexes
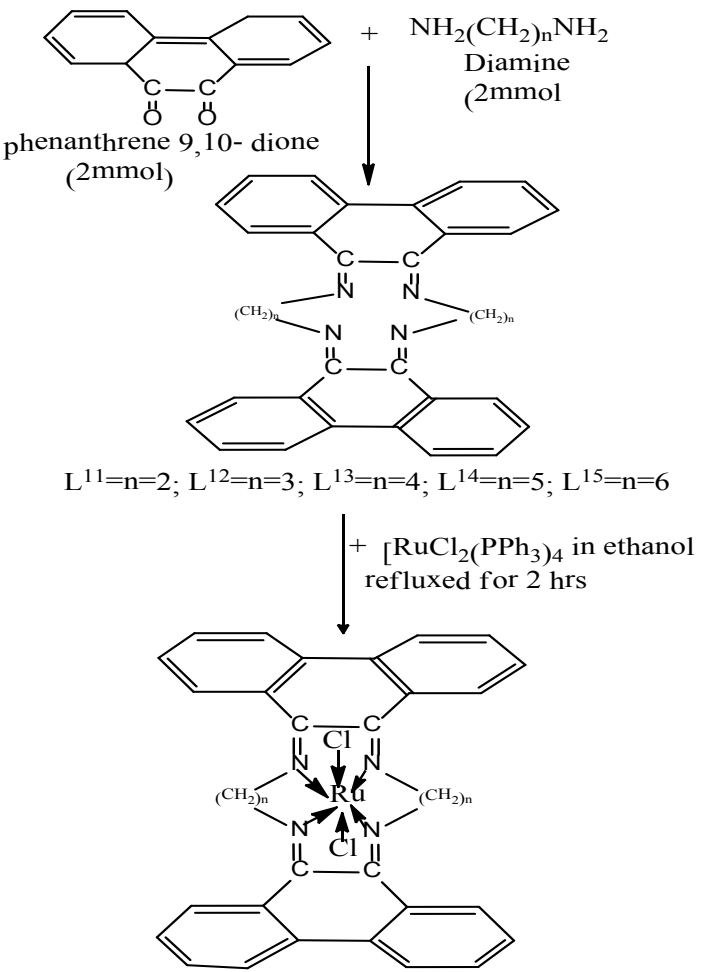

Fig. 3. Preparation of $\left[\mathrm{RuCl}_{2}\left(\mathrm{~L}_{1}{ }^{1-15}\right)\right]$ complexes

RESULTS AND DISCUSSION

Fifteen tetraaza macrocyclic complexes containing $\mathrm{Ru}(\mathrm{II})$ were synthesised by interaction of $\left[\mathrm{RuCl}_{2}\left(\mathrm{PPh}_{3}\right)_{4}\right]$ with fifteen macrocyclic Schiff base ligands $\left(L_{1}\right.$ to $\left.L_{15}\right)$. The complex were soluble in DMF, DMSO and chloroform. The elemental analysis (C, $\mathrm{H}, \mathrm{N}$ and $\mathrm{Cl}$ ) were consistent within $\pm 0.5 \%$ with the suggested structure of the complexes. The molar conductance values were found to below (12.0-20.0 $\mathrm{ohm}^{-1} \mathrm{~cm}^{2} \mathrm{~mol}^{-1}$ ) recommending the non electrolytic nature of the complexes ${ }^{47}$.

In these entire $\left[\mathrm{RuCl}_{2} \mathrm{~L}^{1-15}\right]$ complexes IR band due to $v_{\mathrm{C}=\mathrm{N}}$ was shifted towards lower side about $20-40 \mathrm{~cm}^{-1}$ with respect to the ligand spectra and was obtained in the range of $1600-1580 \mathrm{~cm}^{-1}$. A low intensity band in the region of $520-500 \mathrm{~cm}^{-1}$ were observed due to $v_{\text {Ru-N }}$ vibration confirm that ligands coordinate to Ru metal ion through nitrogen of $\mathrm{C}=\mathrm{N}$ group in all these complexes ${ }^{48}$. Only one band was observed in the range of $320-300 \mathrm{~cm}^{-1}$ in IR spectra of all these complexes indicating the presence of two chloride ions in trans position around ruthenium ion ${ }^{49}$. All the Characteristic IR bands were also observed due to aromatic rings in the expected region in all these $\left[\mathrm{RuCl}_{2} \mathrm{~L}^{1-15}\right]$ complexes ${ }^{49}$. 
The ground state of $\mathrm{Ru}(\mathrm{II}) \mathrm{t}_{2 \mathrm{~g}}{ }^{6}$ electronic configuration is ${ }^{1} \mathrm{~A}_{1 \mathrm{~g}}$. For octahedral $\mathrm{Ru}$ (II) complexes four electronic transition are possible i.e ${ }^{1} A_{1 g} \rightarrow{ }^{3} T_{1 g}$; ${ }^{1} A_{1 g} \rightarrow{ }^{3} T_{2 g} ;{ }^{1} A_{1 g} \rightarrow{ }^{1} T_{1 g}$ and ${ }^{1} A_{1 g} \rightarrow{ }^{1} T_{2 g}$. In each complexes of $\left[\mathrm{RuCl}_{2}\left(\mathrm{~L}^{1-15}\right)\right]$ two electronic bands were observed in the region $200-530 \mathrm{~nm}$. These electronic bands have been assigned to the spin allowed ${ }^{1} \mathrm{~A}_{1 \mathrm{~g}}{ }^{-1} \mathrm{~T}_{1 \mathrm{~g}}$ transition at lower wavelength (460-520 nm) based on molar extinction coefficient.
The another high intensity band at $280-290 \mathrm{~nm}$ region has been assigned due to charge transfer transition originated from the excitation of an electron from the metal ${ }^{\mathrm{t}} \mathrm{g}_{\mathrm{g}}$ level to the unfilled molecular orbital derived from the $\pi^{\star}$ level of the ligands. All these $\left[\mathrm{RuCl}_{2} \mathrm{~L}^{1-15}\right]$ complexes have shown negative magnetic susceptibility and magnetic moment values below then $1.0 \mathrm{BM}$ at room temperature corresponding to diamagnetic nature.

Table 1: Physical and Analytical data of $\left.\left[\mathrm{RuCl}_{2} \mathrm{~L}^{1-15}\right)\right]$ Complexes

\begin{tabular}{|c|c|c|c|c|c|c|}
\hline \multirow[t]{2}{*}{ Sr. No } & \multirow[t]{2}{*}{ Complexes } & \multicolumn{4}{|c|}{$\%$ found (Calculated) } & \multirow[t]{2}{*}{${ }^{\wedge} \mathrm{M} \mathrm{Ohm}^{-1} \mathrm{~cm}^{2} \mathrm{~mol}^{-1}$} \\
\hline & & $\mathrm{C}$ & $\mathrm{H}$ & $\mathrm{N}$ & $\mathrm{Cl}$ & \\
\hline \multirow[t]{2}{*}{1} & $\left.\left[\mathrm{RuCl}_{2} \cdot \mathrm{L}^{1}\right)\right]$ & 41.4 & 3.2 & 11.6 & 15 & 10 \\
\hline & & -41.5 & -3.3 & -11.8 & -15 & \\
\hline \multirow[t]{2}{*}{2} & $\left.\left[\mathrm{RuCl}_{2} \cdot \mathrm{L}^{2}\right)\right]$ & 43.4 & 4 & 11 & 14.2 & 12 \\
\hline & & -43.5 & -4 & -11.2 & -14.3 & \\
\hline \multirow[t]{2}{*}{3} & $\left.\left[\mathrm{RuCl}_{2} \cdot \mathrm{L}^{3}\right)\right]$ & 42.6 & 4.4 & 10.6 & 13.4 & 18 \\
\hline & & -42.7 & -4.5 & -10.6 & -13.5 & \\
\hline \multirow[t]{2}{*}{4} & $\left.\left[\mathrm{RuCl}_{2} \cdot \mathrm{L}^{4}\right)\right]$ & 47.6 & 5 & 10 & 12.6 & 16 \\
\hline & & -47.8 & -5 & -10.1 & -12.8 & \\
\hline \multirow[t]{2}{*}{5} & $\left.\left[\mathrm{RuCl}_{2} \cdot \mathrm{L}^{5}\right)\right]$ & 50.4 & 5.4 & 9.4 & 12 & 10 \\
\hline & & -50.5 & -5.4 & -9.4 & -12 & \\
\hline \multirow[t]{2}{*}{6} & $\left.\left[\mathrm{RuCl}_{2} \cdot \mathrm{L}^{6}\right)\right]$ & 54.2 & 3.6 & 10.5 & 13.4 & 12 \\
\hline & & -54.4 & -3.7 & -10.5 & -13.4 & \\
\hline \multirow[t]{2}{*}{7} & $\left.\left[\mathrm{RuCl}_{2} \cdot \mathrm{L}^{7}\right)\right]$ & 56 & 4.2 & 10 & 12.6 & 14 \\
\hline & & -56 & -4.3 & -10 & -12.7 & \\
\hline \multirow[t]{2}{*}{8} & $\left.\left[\mathrm{RuCl}_{2} \cdot \mathrm{L}^{8}\right)\right]$ & 69 & 5.6 & 11.4 & 14.6 & 16 \\
\hline & & -69.2 & -5.7 & -11.5 & -14.6 & \\
\hline \multirow[t]{2}{*}{9} & $\left.\left[\mathrm{RuCl}_{2} \cdot \mathrm{L}^{9}\right)\right]$ & 30.6 & 4 & 7 & 9 & 12 \\
\hline & & -30.7 & -4.4 & -7.1 & -9 & \\
\hline \multirow[t]{2}{*}{10} & $\left.\left[\mathrm{RuCl}_{2} \cdot \mathrm{L}^{10}\right)\right]$ & 59.8 & 3.2 & 11.6 & 15 & 18 \\
\hline & & -59.9 & -3.3 & -11.8 & -15 & \\
\hline \multirow[t]{2}{*}{11} & $\left.\left[\mathrm{RuCl}_{2} \cdot \mathrm{L}^{11}\right)\right]$ & 68 & 4.8 & 7 & 9 & 20 \\
\hline & & -68 & -4.9 & -7.1 & -9 & \\
\hline \multirow[t]{2}{*}{12} & $\left.\left[\mathrm{RuCl}_{2} \cdot \mathrm{L}^{12}\right)\right]$ & 68.6 & 4.6 & 9.2 & 11.8 & 18 \\
\hline & & -68.8 & -4.7 & -9.4 & -11.9 & \\
\hline \multirow[t]{2}{*}{13} & $\left.\left[\mathrm{RuCl}_{2} \cdot \mathrm{L}^{13}\right)\right]$ & 69.4 & 5 & 9 & 11.2 & 16 \\
\hline & & -69.5 & -5.1 & -9 & -11.4 & \\
\hline \multirow[t]{2}{*}{14} & $\left.\left[\mathrm{RuCl}_{2} \cdot \mathrm{L}^{14}\right)\right]$ & 71.2 & 4 & 8.6 & 11 & 14 \\
\hline & & -71.3 & -4.4 & -8.7 & -11.1 & \\
\hline \multirow[t]{2}{*}{15} & $\left.\left[\mathrm{RuCl}_{2} \cdot \mathrm{L}^{15}\right)\right]$ & 75 & 4.2 & 8.6 & 11 & 12 \\
\hline & & -75.1 & -4.3 & -8.7 & -11.1 & \\
\hline
\end{tabular}

Table 2: Infrared Spectral data of $\left[\mathrm{RuCl}_{2}\left(\mathrm{~L}^{1-15}\right)\right]$ Complexes

\begin{tabular}{ccccc}
\hline Sr. No & Complex & \multicolumn{3}{c}{ Selected IR bands $\left(\mathrm{cm}^{-1}\right)$} \\
& & $\mathrm{V}_{\mathrm{C}=\mathrm{N}}$ & $\mathrm{V}_{\text {Ru-N }}$ & $\mathrm{V}_{\text {Ru-Cl }}$ \\
\hline 1 & $\left.\left[\mathrm{RuCl}_{2} \cdot \mathrm{L}^{1}\right)\right]$ & 1580 & 520 & 320 \\
2 & $\left.\left[\mathrm{RuCl}_{2} \cdot \mathrm{L}^{2}\right)\right]$ & 1570 & 515 & 310 \\
3 & $\left.\left[\mathrm{RuCl}_{2} \cdot \mathrm{L}^{3}\right)\right]$ & 1575 & 518 & 305 \\
4 & $\left.\left[\mathrm{RuCl}_{2} \cdot \mathrm{L}_{4}\right)\right]$ & 1580 & 515 & 316 \\
5 & $\left.\left[\mathrm{RuCl}_{2} \cdot \mathrm{L}_{5}\right)\right]$ & 1570 & 500 & 300 \\
6 & $\left.\left[\mathrm{RuCl}_{2} \cdot \mathrm{L}_{6}\right)\right]$ & 1580 & 510 & 315 \\
7 & $\left.\left[\mathrm{RuCl}_{2} \cdot \mathrm{L}_{7}\right)\right]$ & 1580 & 505 & 305 \\
8 & $\left.\left[\mathrm{RuCl}_{2} \cdot \mathrm{L}_{8}\right)\right]$ & 1580 & 500 & 320 \\
9 & $\left.\left[\mathrm{RuCl}_{2} \cdot \mathrm{L}_{9}\right)\right]$ & 1600 & 515 & 315 \\
10 & $\left.\left[\mathrm{RuCl}_{2} \cdot \mathrm{L}_{10}\right)\right]$ & 1600 & 518 & 320 \\
11 & $\left.\left[\mathrm{RuCl}_{2} \cdot \mathrm{L}_{11}\right)\right]$ & 1590 & 510 & 320 \\
12 & $\left.\left[\mathrm{RuCl}_{2} \cdot \mathrm{L}_{12}\right)\right]$ & 1580 & 508 & 315 \\
13 & $\left.\left[\mathrm{RuCl}_{2} \cdot \mathrm{L}_{13}\right)\right]$ & 1600 & 515 & 310 \\
14 & $\left.\left[\mathrm{RuCl}_{2} \cdot \mathrm{L}_{14}\right)\right]$ & 1580 & 520 & 305 \\
15 & $\left.\left[\mathrm{RuCl}_{2} \cdot \mathrm{L}_{15}\right)\right]$ & 1580 & 515 & 320 \\
\hline
\end{tabular}


Table 3: Ru3p $p_{1 / 2,3 / 2} ; N_{1 s}$ and $\mathrm{Cl}_{2 p}$ binding energies $(\mathrm{eV})$ in ligand; $\left[\mathrm{RuCl}_{2}\left(\mathrm{~L}^{1-15}\right)\right]$ complexes

\begin{tabular}{|c|c|c|c|c|c|}
\hline \multirow[t]{2}{*}{ Sr. No } & \multirow[t]{2}{*}{ Ligand \& Complexes } & \multicolumn{2}{|c|}{$\operatorname{Ru}_{1 / 2,3 / 2}$} & \multirow[t]{2}{*}{$\mathrm{N}_{1 \mathrm{~s}}$} & \multirow[t]{2}{*}{$\mathrm{Cl}_{2 \mathrm{p}}$} \\
\hline & & ${\operatorname{Ru} 3 P_{1 / 2}}_{1}$ & $\mathrm{Ru} \mathrm{P}_{3 / 2}$ & & \\
\hline 1 & $\mathrm{~L}_{1}$ & --- & --- & 400.6 & --- \\
\hline 2 & $\mathrm{~L}_{2}$ & --- & --- & 400.6 & --- \\
\hline 3 & $\mathrm{~L}_{3}$ & --- & --- & 400.6 & --- \\
\hline 4 & $\mathrm{~L}_{4}^{3}$ & --- & --- & 400.6 & --- \\
\hline 5 & $\mathrm{~L}_{5}$ & --- & --- & 400.6 & --- \\
\hline 6 & $L_{6}$ & --- & --- & 400.6 & --- \\
\hline 7 & $\mathrm{~L}_{7}$ & --- & --- & 400.6 & --- \\
\hline 8 & $\mathrm{~L}_{8}$ & --- & --- & 400.6 & --- \\
\hline 9 & $\mathrm{~L}_{9}$ & --- & --- & 400.6 & --- \\
\hline 10 & $L_{11}$ & --- & --- & 400.6 & --- \\
\hline 11 & $L_{11}$ & --- & --- & 400.6 & --- \\
\hline 12 & $L_{12}^{\prime \prime}$ & --- & --- & 400.6 & --- \\
\hline 13 & $\mathrm{~L}_{13}$ & --- & --- & 400.6 & --- \\
\hline 14 & $\mathrm{~L}_{14}$ & --- & --- & 400.6 & --- \\
\hline 15 & $L_{15}^{14}$ & --- & --- & 400.6 & --- \\
\hline 16 & {$\left[\mathrm{RuCl}_{2}\left(\mathrm{PPh}_{3}\right)_{4}\right]$} & 482.8 & 460.8 & --- & 201.6 \\
\hline 17 & $\left.\left[\operatorname{RuCl}_{2} \cdot \mathrm{L}^{1}\right)\right]$ & 482 & 460 & 402.8 & 202.8 \\
\hline 18 & $\left.\left[\mathrm{RuCl}_{2} \cdot \mathrm{L}^{2}\right)\right]$ & 482 & 460 & 402.8 & 202.8 \\
\hline 19 & $\left.\left[\mathrm{RuCl}_{2} \cdot \mathrm{L}^{3}\right)\right]$ & 482 & 460 & 402.8 & 202.8 \\
\hline 20 & $\left.\left[\mathrm{RuCl}_{2} \cdot \mathrm{L}^{4}\right)\right]$ & 482 & 460 & 402.8 & 202.8 \\
\hline 21 & $\left.\left[\mathrm{RuCl}_{2} \cdot \mathrm{L}^{5}\right)\right]$ & 482 & 460 & 402.8 & 202.8 \\
\hline 22 & $\left.\left[\operatorname{RuCl}_{2}^{2} \cdot L^{6}\right)\right]$ & 482 & 460 & 402.8 & 202.8 \\
\hline 23 & $\left.\left[\mathrm{RuCl}_{2} \cdot \mathrm{L}^{7}\right)\right]$ & 482 & 460 & 402.8 & 202.8 \\
\hline 24 & $\left.\left[\mathrm{RuCl}_{2} \cdot \mathrm{L}^{8}\right)\right]$ & 482 & 460 & 402.8 & 202.8 \\
\hline 25 & $\left.\left[\operatorname{RuCl}_{2} \cdot \mathrm{L}^{9}\right)\right]$ & 482 & 460 & 402.8 & 202.8 \\
\hline 26 & $\left.\left[\mathrm{RuCl}_{2} \cdot \mathrm{L}^{10}\right)\right]$ & 482 & 460 & 402.8 & 202.8 \\
\hline 27 & $\left.\left[\mathrm{RuCl}_{2} \cdot \mathrm{L}^{11}\right)\right]$ & 482 & 460 & 402.8 & 202.8 \\
\hline 28 & $\left.\left[\mathrm{RuCl}_{2} \cdot \mathrm{L}^{12}\right)\right]$ & 482 & 460 & 402.8 & 202.8 \\
\hline 29 & $\left.\left[\mathrm{RuCl}_{2} \cdot \mathrm{L}^{13}\right)\right]$ & 482 & 460 & 402.8 & 202.8 \\
\hline 30 & $\left.\left[\mathrm{RuCl}_{2} \cdot \mathrm{L}^{14}\right)\right]$ & 482 & 460 & 402.8 & 202.8 \\
\hline 31 & $\left.\left[\mathrm{RuCl}_{2} \cdot \mathrm{L}^{15}\right)\right]$ & 482 & 460 & 402.8 & 202.8 \\
\hline
\end{tabular}

The binding energies $(\mathrm{eV})$ of prepared ligands i.e. $\mathrm{L}^{1-15} ;\left[\mathrm{RuCl}_{2}\left(\mathrm{PPh}_{3}\right)_{4}\right]$ and $\left[\mathrm{RuCl}_{2}\left(\mathrm{~L}^{1-15}\right)\right]$ in Table 1 (Figs. 4-6) for Ru3p $p_{1 / 2,3 / 2}, N_{1 s}$ and $\mathrm{Cl} 2 \mathrm{p}$ photoelectron peaks. It was observed that Ru3p $\mathrm{p}_{1 / 2,3 / 2}$ binding energies in starting material $\left[\mathrm{RuCl}_{2}\left(\mathrm{PPh}_{3}\right)_{4}\right]$ were higher $(\mathrm{BE}=\sim 482.8 \mathrm{eV}$ and

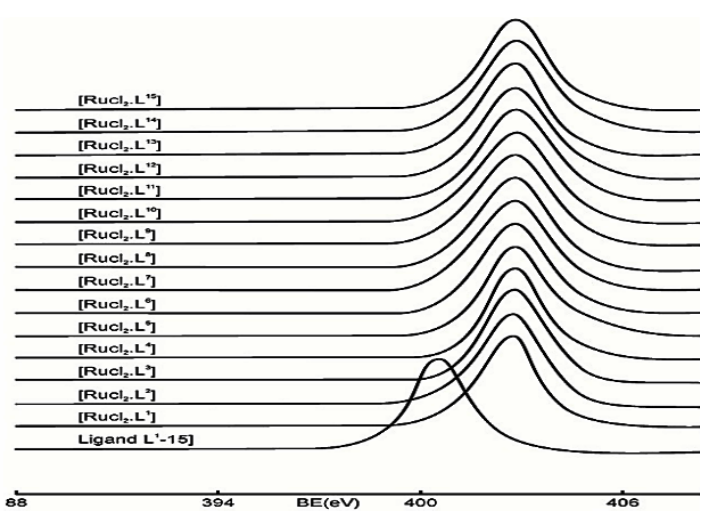

Fig. 4. Ru3 $p_{1 / 2}$ binding energies $(\mathrm{eV})$ in $\left[\mathrm{RuCl}_{2}\left(\mathrm{~L}^{1-15}\right)\right]$ complexes
$460.8 \mathrm{eV}$ for Ru3p $\mathrm{p}_{1 / 2}$ and Ru3p $\mathrm{p}_{3 / 2}$ ) than in prepared $\left[\mathrm{RuCl}_{2} \mathrm{~L}^{1-15}\right]$ complexes (Ru3p ${ }_{1 / 2} \mathrm{BE}=482.0 \mathrm{eV}$ and $\mathrm{Ru} \mathrm{P}_{3 / 2} \mathrm{BE} 460.0 \mathrm{eV}$ ); suggesting that electron density in ruthenium metal ion is more in prepared $\left[\mathrm{RuCl}_{2} \mathrm{~L}^{1-15}\right]$ complexes than in $\left[\mathrm{RuCl}_{2}\left(\mathrm{PPh}_{3}\right)_{4}\right]$ due to coordination $^{50}$ (Figures 4-6).

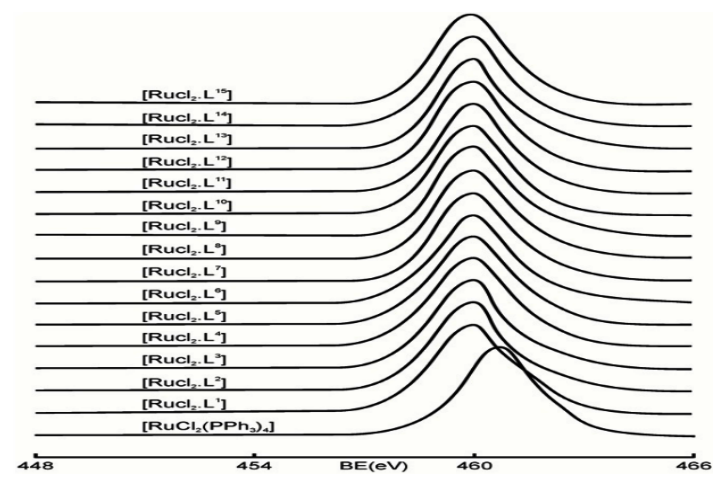

Fig. 5. $\mathrm{Ru}_{2} \mathrm{p}^{3 / 2}$ binding energies $(\mathrm{eV})$ in $\left[\mathrm{RuCl}_{2}\left(\mathrm{~L}^{1-16}\right)\right]$ complexes prepared $\left[\mathrm{RuCl}_{2}\left(\mathrm{~L}^{1-15}\right)\right]$ metal complexes 


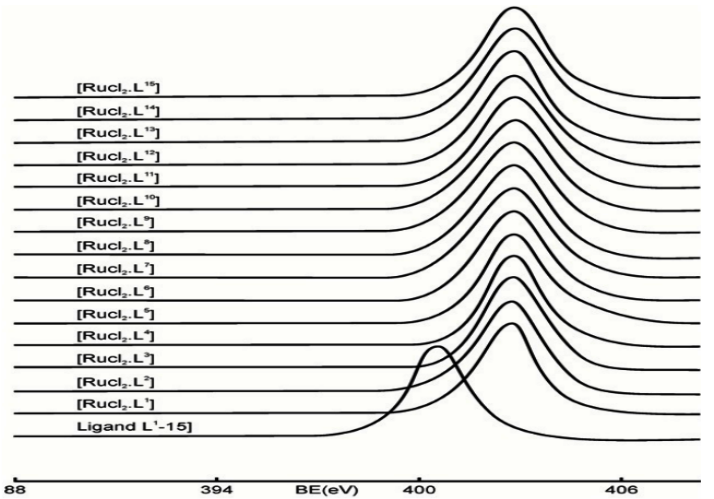

Fig. 6. $\mathrm{N}_{1 \mathrm{~s}}$ binding energies $(\mathrm{eV})$ in Ligands and $\left[\mathrm{RuCl}_{2}\left(\mathrm{~L}^{1-15}\right)\right]$ complexes prepared

The $\mathrm{N}_{1 \mathrm{~s}}$ photoelectron spectra of all these $\left[\mathrm{RuCl}_{2}\left(\mathrm{~L}^{1-15}\right)\right]$ complexes have shown only one signal symmetrical photoelectron peak towards higher binding energies side ( $\mathrm{BE}=\sim 402.8 \mathrm{eV}$ ) than N1S photoelectron peak of each ligand $(\sim \mathrm{BE}=400.6$ eV) suggesting all four nitrogen atoms of each ligand is coordinated with ruthenium (II) metal ion..$^{50}$ (Fig. 6) \& Table 1) The $\mathrm{Ru}_{3} \mathrm{~S}$ photoelectron peak in all these prepared $\left[\mathrm{RuCl}_{2}\left(\mathrm{~L}^{1-15}\right]\right.$ metal complexes. have shown symmetrical peak at 584.6 i.e. have not shown multiple splitting diamagnetic nature ${ }^{50}$. The $\mathrm{Cl}_{2 p}$ photoelectron spectra of all these $\left[\mathrm{RuL}_{2}\left(\mathrm{~L}^{1-15}\right]\right.$ complexes have shown one sharp peak at BE
202.4-202.0 eV; suggesting inner sphere chlorine atom in trans position ${ }^{50}$.

\section{CONCLUSION}

In the present paper, fiffteen $\mathrm{Ru}(\mathrm{II})$ complexes of the type $\left[\mathrm{RuCl}_{2} \mathrm{~L}^{1-15}\right]$ have been synthesized and characterized with fifteen tetraaza macrocyclic ligands. On account of analytical and spectral statistics octahedral geometry were provisionally proposed for all of these complexes.

\section{ACKNOWLEDGEMENT}

The author Ashish Rajak is thankful to Chemistry Department, University of Allahabad, Prayagraj for affording financial assistance to the research work and instrumental facilities and Arpit Srivastava, Ramakant and SCS are gratified to UGCCSIR, New Delhi, India for providing the financial support as UGC JRF (Ref. no-191620091263), UGC-SRF (Ref. no-354/CSIR-UGC NET DEC. 2017) and UGC-SRF (Ref. no-19/06/2016(i)EU-V) respectively.

\section{Conflicts of interest}

The authors declare no conflict of interest in the present research work.

\section{REFERENCES}

1. Kumar, R.; Singh, R. Russ J Coord Chem., 2006, 32(3), 192-198.

2. Chaudhary, A.; Bansal, N.; Gajraj, A.; Singh, R.V. J inorg biochem., 2003, 96(2-3), 393-400.

3. Zhong, Z.J.; You, X.Z.; Mak, T.C. Polyhedron., 1994, 13(14), 2157-2161.

4. Reddy, P.M.; Ho, Y.P; Shanker, K.; Rohini, R. Eur J Med chem., 2009, 44(6), 2621-2625.

5. Reddy, P.M.; Prasad, A.V; Shanker, K.; Ravinder, V. Spectrochim Acta A Mol Biomol Spectrosc., 2007, 68(3), 1000-1006.

6. Reddy, P.M.; Prasad, A.V.; Rohini, R.; Ravinder, V. Spectrochim Acta A Mol Biomol Spectrosc., 2008, 70(3), 704-712.

7. Akine, S.; Sunaga, S.; Taniguchi, T.; Miyazaki, H.; Nabeshima, T. Inorg Chem., 2007, 46(8), 2959-2961.

8. Anacona, J.R.; Bastardo, E.; Camus, J. Transit Met Chem., 1999, 24(4), 478-480.

9. Nazmutdinov, R.R.; Roznyatovskaya, N.V.;
Glukhov, D.V.; Manyurov, I.; Mazin, V.M.; Tsirlina, G.A.; Probst, M. Inorg Chem., 2008, 47(15), 6659-6673.

10. Hodacová, J.; Buděšínský, M. Org Lett., 2007, 9(26), 5641-5643.

11. Constable, E.C.; King, A.C.; Palmer, C.A. Inorganica Chim Acta., 1991, 184(1), 43-45.

12. Gokel, G.W.; Leevy, W.M.; Weber, M.E. Chem Rev., 2004, 104(5), 2723-2750.

13. López-Deber, M.; Bastida de la Calle, R.; Macías, A.; Pérez-Lourido, P.; Rodríguez, A.; Valencia Matarranz, L. Z Anorg Allg Chem., 2007, 633(11-12), 1842-1846.

14. Bértolo, E.; Bastida, R.; De Blas, A.; Fenton, D.E.; Lodeiro, C.; Macías, A.; Rodríguez, A.; Rodríguez-Blas, T. J Incl Phenom Macrocycl Chem., 1999, 35(1), 191-198.

15. Salavati-Niasari, M.; Davar, F. Inorg Chem Commun., 2006, 9(2), 175-179.

16. Saleh, A.A. J Coord Chem., 2005, 58(3), 255-270. 
17. Emara, A.A.; Abou-Hussen, A.A. Spectrochim Acta A Mol Biomol Spectrosc., 2006, 64(4), 1010-1024.

18. Kosmas, C.; Snook, D.; Gooden, C.; Courtenay-Luck, N.S.; McCall, M.J.; Meares, C.F.; Epenetos, A.A. Cancer Res., 1992, 52(4), 904-911.

19. Mewis, R.E.; Archibald, S. J. Coord Chem Rev., 2010, 254(15-16), 1686-1712.

20. Adam, K.R.; Antolovich, M.; Baldwin, D.S.; Duckworth, P.A.; Leong, A.J.; Lindoy, L.F.; McPartlin, M.; Tasker, P.A. J Chem Soc., Dalton Trans., 1993, 1(7), 1013-1017.

21. El-Boraey, H.A.; El-Gammal, O.A. Spectrochim Acta A Mol Biomol Spectrosc., 2015, 138, 553-62.

22. Tyagi, M.; Chandra, S.; Akhtar, J.; Chand, D. Spectrochim Acta A Mol Biomol Spectrosc., 2014, 118, 1056-1061.

23. Bonnett, R. Chem Soc Rev., 1995, 24(1), 19-33.

24. Champness, N.R.; Frampton, C.S.; Reid, G.; Tocher, D. J Chem Soc., Dalton Trans., 1994, 20, 3031-3037.

25. Makki, M.S.; Abdel-Rahman, R.M.; ElShahawi, M.S. Comptes Rendus Chimie., 2012, 15(7), 617-626.

26. Canales. J.; Ramirez, J.; Estiu, G.; Costamagna, J. Polyhedron., 2000, 19(22-23), 2373-2381.

27. Bencini, A.; Bianchi, A.; Giorgi, C.; Paoletti, P.; Valtancoli, B.; Fusi, V.; García-España, E.; Llinares, J.M.; Ramírez, J. A. Inorg Chem., 1996, 35(5), 1114-1120.

28. Singh, R.V.; Dwivedi, R.; Joshi, S.C. Transit Met Chem., 2004, 29(1), 70-74.

29. Jain, M.; Gaur, S.; Diwedi, S.C.; Joshi, S.C.; Singh. R.V.; Bansal, A. Phosphorus, Sulfur, and Silicon and the Related Elements., 2004, 179(8), 1517-1537.

30. Maldonado, C.R.; Salassa, L.; GomezBlanco, N.; Mareque-Rivas, J. C. Coord Chem Rev., 2013, 257(19-20), 2668-2688.

31. Majkowska-Pilip, A.; Bilewicz, A. J Inorg Biochem., 2011, 105(2), 313-320.

32. El-Boraey, H.A.; El-Din, A.A. Spectrochim Acta A Mol Biomol Spectrosc., 2014, 132,
663-671.

33. Blain, S.; Appriou, P.; Chaumeil, H.; Handel, H. Analytica Chim Acta., 1990, 232, 331-336.

34. Chaudhary, A.; Singh, R.V. Phosphorus, Sulfur, and Silicon and the Related Elements., 2007, 182(11), 2647-2665.

35. Buster, D.C.; Castro, M.M.; Geraldes, C.F.; Malloy, C.R.; Sherry, AD.; Siemers, T.C. Magnetic resonance in medicine., 1990, 15(1), 25-32.

36. Choi, K.Y.; Chun, K.M.; Suh, I.H. Polyhedron., 2001, 20(1-2), 57-65.

37. Baker, B.F.; Khalili, H.; Wei, N.; Morrow, J.R.; JAm Chem Soc., 1997, 119(38), 8749-8755.

38. Buster, D.C.; Gaughan G.T.; Hagan, J.N. U.S. Patent 4885363; 1987.

39. Ochiai E. Coord Chem Rev., 2010, 254 (15-16), 1812-1814.

40. Prabhakaran, R.; Geetha, A.; Thilagavathi, M.; Karvembu, R.; Krishnan, V.; Bertagnolli, H.; Natarajan, K. J Inorg biochem., 2004, 98(12), 2131-2140.

41. Wong, C.Y.; Lee, F.W.; Che, C.M.; Cheng, Y.F.; Phillips, D.L.; Zhu, N. Inorg Chem., 2008, 47(22), 10308-10316.

42. Shanker, K.; Reddy, P.; Ravinder, V. Int J Chem Tech Res., 2009, 1, 300-307.

43. Doro, F.G.; Ferreira, K.Q.; da Rocha, Z.N.; Caramori, G.F., Gomes, A.J.; T founi, E. Coord Chem Rev., 2016, 306, 652-677.

44. Tfouni, E.; Ferreira, K.Q.; Doro, F.G.; da Silva, R.S.; da Rocha, Z.N. Coord Chem Rev., 2005, 249(3-4), 405-418.

45. Rawling, T.; McDonagh, A. Coord Chem Rev., 2007, 251(9-10), 1128-1157.

46. Shanker, K.; Reddy, P.; Ravinder, V. Int J Chem Tech Res., 2009, 1, 300-307.

47. Jolly, W.L. Coord Chem Rev., 1974, 13(1), 47-81.

48. Shakir, M.; Nasman, O.S.; Varkey, S.P. Polyhedron., 1996, 15(2), 309-314.

49. Reddy, P.M.; Prasad, A.V.; Shanker, K.; Ravinder, V. Spectrochim Acta A Mol Biomol Spectrosc., 2007, 68(3), 1000-1006.

50. Sriastava S. Appl Spectrosc Rev., 1986, 22(4), 401-535. 